\title{
Primer registro de tres especies de peces en el Río Grande de San Ramón, Costa Rica
}

\author{
Cindy Rodríguez-Arias ${ }^{1}$, Marco Zumbado Echavarría ${ }^{2}$ \& David Alfaro Chaves ${ }^{3}$ \\ 1. Universidad de Costa Rica, Sede de Occidente, Sección de Biología, San Ramón, Alajuela, Costa Rica; cindy.rodriguez_a@ucr.ac.cr, \\ (iD) https://orcid.org/0000-0002-4074-8094 \\ 2. Universidad Costa Rica, Sede de Occidente, Laboratorio de Biología, San Ramón, Alajuela, Costa Rica; marco.zumbado@ucr.ac.cr, \\ (D) https://orcid.org/0000-0002-2591-7662 \\ 3. Universidad Costa Rica, Sede de Occidente, Estudiante de la carrera de Gestión de Recursos Naturales, San Ramón, Alajuela, Costa Rica; \\ josedavid201187@hotmail.com, (D) https://orcid.org/0000-0001-7299-9739
}

Recibido 01-II-2019 • Corregido 10-VI-2019 • Aceptado 13-VI-2019

DOI: https://doi.org/10.22458/urj.v11i3.2608

\begin{abstract}
First record of three fish species in Río Grande de San Ramón, Costa Rica". Four sampling trips (2016 and 2017) in Río Grande de San Ramón (Tárcoles River Basin, 1 060masl) produced nine species, three of them for the first time for this altitude or basin. Previously, Alfaro cultratus ( $\mathrm{N}=95)$ was reported only up to 300 masl at the Caribbean slope and in the Tempisque River basin; Amatitlania siquia $(\mathrm{N}=4)$ only under 540masl for the Tárcoles river basin; and Amatitlania septemfasciata $(\mathrm{N}=22)$ from the Atlantic slope and below 600 masl.
\end{abstract}

Key words: distribution area, freshwater fishes, Alfaro cultratus, Amatitlania siquia, Amatitlania septemfasciata.
RESUMEN: Registramos nueve especies de peces durante cuatro giras de muestreo (2016 y 2017) en el Río Grande de San Ramón (cuenca del río Tárcoles, $1060 \mathrm{msnm}$ ), tres de ellas por primera vez para esta altitud o cuenca. Previamente, Alfaro cultratus ( $\mathrm{N}=95)$ solo se informaba hasta $300 \mathrm{msnm}$ en la vertiente caribeña y en la cuenca del río Tempisque; Amatitlania siquia $(\mathrm{N}=4)$ solo por debajo de $540 \mathrm{msnm}$ para la cuenca del río Tárcoles; y Amatitlania septemfasciata $(\mathrm{N}=22)$ desde la vertiente atlántica y por debajo de $600 \mathrm{msnm}$.

Palabras clave: distribución geográfica, peces de agua dulce, Alfaro cultratus, Amatitlania siquia, Amatitlania septemfasciata.
A pesar de su pequeño tamaño continental, Costa Rica tiene una rica fauna de peces de agua dulce y el conocimiento sobre nuevas especies y registros en nuevas localidades continúa creciendo (Angulo, Garita-Alvarado, Bussing, \& López, 2013). Además, es importante documentar esta riqueza debido a los rápidos cambios que las actividades antrópicas están causando en los ríos (Bussing, 2002) y a los impactos ambientales negativos a los que están expuestos (Rojas \& Rodríguez, 2008).

Por esta razón, durante los años 2016 y 2017 se desarrolló una investigación en la parte alta de la microcuenca de la quebrada Estero en San Ramón de Alajuela, Costa Rica (Rodríguez \& Zumbado, 2018), en la cual se estableció como ecosistema de referencia un sector del Río Grande de San Ramón (cuenca alta del Río Tárcoles). Como parte de los resultados en dicho río se encontraron tres especies que no estaban reportadas previamente en la literatura, por lo que el objetivo de este documento es contribuir al conocimiento de la distribución geográfica de estos peces y de la ictiofauna en general del Río Grande de San Ramón.

Se realizaron cuatro muestreos en noviembre del 2016, enero, marzo y agosto del 2017, a lo largo de un transecto ubicado entre las coordenadas $10^{\circ} 03^{\prime} 35,1^{\prime \prime} \mathrm{N}-84^{\circ} 29^{\prime} 13,9^{\prime \prime} \mathrm{W}$ y $10^{\circ} 03^{\prime} 54,5^{\prime \prime} \mathrm{N}-84^{\circ} 28^{\prime} 50,4^{\prime \prime} \mathrm{W}$ en el distrito de Santiago y a una altitud media de 1 060msnm. Para la colecta de los peces se utilizó una atarraya marca Silkdia de $0,35 \mathrm{~mm}$, maya de $1 / 2$ " y profundidad de 4". Las capturas se realizaron en pozas, aguas rápidas, aguas estancadas, aguas profundas y aguas bajas, durante $95 \mathrm{~min}$. También se colocaron trampas de botellas perforadas con carnada durante $95 \mathrm{~min}$. La mayoría de los individuos capturados fueron liberados tras su identificación, mientras que algunos fueron colectados para su mejor estudio en el Laboratorio de Biología de la Sede de Occidente de la Universidad de Costa Rica (UCR). Las muestras de las especies que no se habían reportado previamente en este río fueron depositadas en el Museo 
de Zoología de la UCR. Para la identificación de las especies se utilizaron las claves taxonómicas de Bussing (2002) y la taxonomía según Fricke, Eschmeyer, y Van der Laan (2019).

Se encontraron cuatro familias y nueve especies de peces, todas nativas y una endémica de Costa Rica (Cuadro 1). No se encontraron publicaciones previas sobre la ictiofauna del Río Grande, sin embargo, Bussing (2002) y Angulo, Garita-Alvarado, Bussing, y López (2013), mencionan la presencia de ocho especies nativas y cuatro exóticas por encima de los $1000 \mathrm{msnm}$ en la cuenca del río Tárcoles. De éstas, seis especies coinciden con lo encontrado en el presente estudio y las que no se reportaron fueron Rhamdia nicaraguensis (Günther, 1864), Cynodonichthys isthmensis (Garman, 1895), Priapichthys annectens (Regan, 1907) y las exóticas Poecilia reticulata, Carassius auratus, Cyprinus carpio y Oreochromis niloticus. Por otro lado, fue posible capturar tres especies que no estaban registradas previamente para esta altitud y/o esta cuenca: Alfaro cultratus, Amatitlania siquia y Amatitlania septemfasciata.

La especie Alfaro cultratus (Fig. 1), conocida como olomina de Alfaro (Angulo, 2013) estaba reportada solamente para altitudes entre 0 y $300 \mathrm{msnm}$ de la vertiente Caribe y como especie poco común en la cuenca del Tempisque (Bussing, 2002; Angulo et al., 2013). Por lo tanto, este registro permite ampliar su ámbito de distribución a una nueva cuenca y a una altitud mayor.

En el sitio de estudio fue capturada en los cuatro muestreos, con un total de 95 individuos, en pozas de alrededor de un metro de profundidad y corrientes lentas, con fondo arenoso y presencia de hojarasca. Su identificación fue posible debido a que es la única especie de esta familia que presenta una quilla en el margen inferior, formada por el margen ventral del pedúnculo caudal afilado y comprimido (Bussing, 2002). Se depositó una muestra de esta especie en el Museo de Zoología de la UCR bajo el número de registro UCR3297002.

Las otras dos especies pertenecen a la familia Cichlidae. La mojarra rayada o convicto Amatitlania siquia (Fig. 2), estaba reportada para la cuenca del Río Tárcoles, pero solamente para altitudes entre 0 y $540 \mathrm{msnm}$ (Bussing, 2002; Angulo et al., 2013), mientras que en el presente estudio se encontró por encima de los $1000 \mathrm{msnm}$, por lo que se considera importante contribuir al conocimiento de su distribución actual.

De esta especie se capturaron dos individuos en enero y dos en agosto del 2017, en zonas de corriente de media a alta con fondo pedregoso y unos $40 \mathrm{~cm}$ de profundidad, así como en pozas de $1 \mathrm{~m}$ de profundidad con fondo arenoso y presencia de hojarasca. Las características mencionadas por Bussing (2002) que permitieron su identificación fueron: el iris de color dorado, la mancha oscura en el opérculo, siete barras en total, la última en la base de la cola y la primera barra en forma de Y (Fig. 2). Se depositó una muestra de esta especie en el Museo de Zoología de la UCR con el número de registro UCR3298001.

Por su parte, la mojarra de la especie Amatitlania septemfasciata (Fig. 3) había sido registrada solamente en la vertiente atlántica y en altitudes entre 12 y $600 \mathrm{msnm}$ (Bussing, 2002, Angulo et al. 2013), por lo que es la primera vez que se reporta en la vertiente Pacífica y a una altitud superior a los $1000 \mathrm{msnm}$.

Se logró identificar gracias a características como el iris de color azul, la mancha lateral ovalada sobre la tercera barra lateral, seis barras sobre el cuerpo, que son tenues en los adultos, y una sobre el pedúnculo caudal. También

CUADRO 1

Familias y especies de peces capturadas en el Río Grande de San Ramón, Costa Rica

\begin{tabular}{llc}
\multicolumn{1}{c}{ Familia } & \multicolumn{1}{c}{ Especie } & Nombre común \\
Characidae & Astyanax aeneus (Günther, 1860) & Sardina \\
Heptapteridae & Rhamdia laticauda (Kner, 1858) & Barbudo \\
Poeciliidae & Alfaro cultratus (Regan, 1908) & Olomina, Alfaro \\
& Brachyrhaphis olomina (Meek, 1914)* & Olomina \\
& Phallichthys amates (Miller, 1907) & Olomina \\
& Poecilia gillii (Kner,1863) & Olomina \\
Cichlidae & Amatitlania siquia (Schmitter-Soto, 2007) & Mojarra \\
& Cribroheros alfari (Meek, 1907) & Mojarra \\
& Amatitlania septemfasciata (Regan, 1908) & Mojarra \\
\hline
\end{tabular}

* Especie endémica de Costa Rica. 

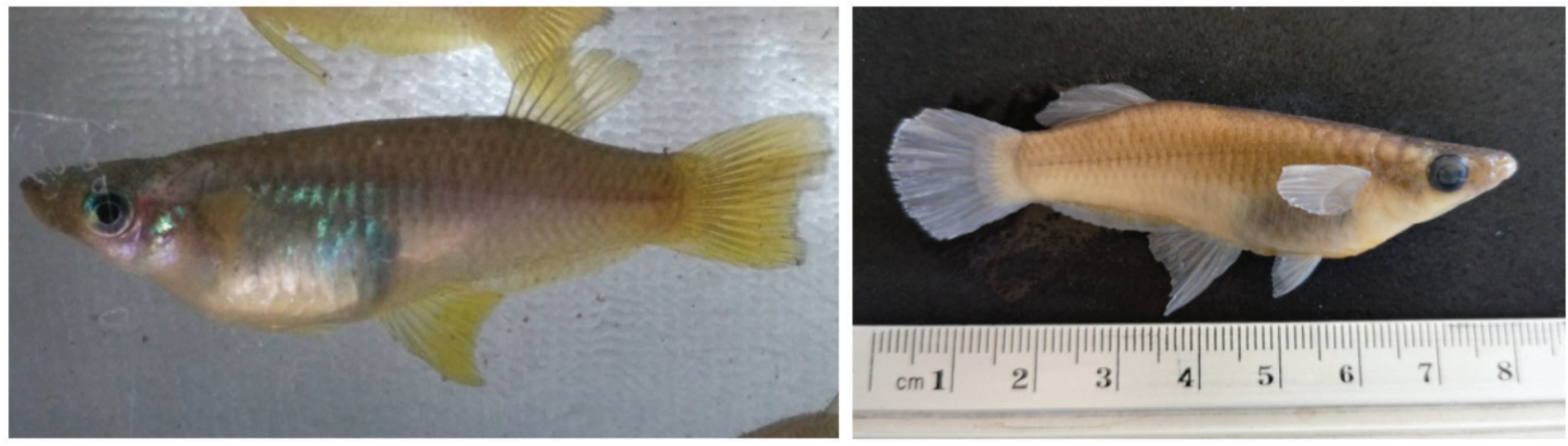

Fig. 1. Individuo de A. cultratus. Se observa, como característica diagnóstica la quilla en el margen inferior del pedúnculo caudal.
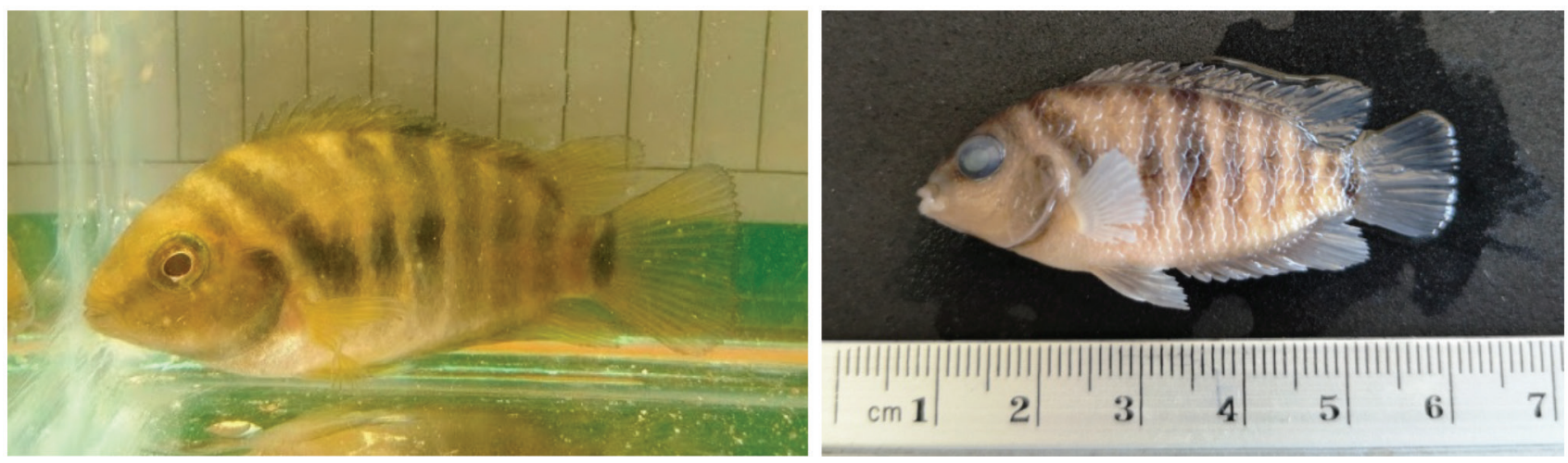

Fig. 2. Individuo de A. siquia. Se observan como características diagnósticas la mancha negra en el opérculo, las seis barras negras en el cuerpo, la primera en forma de Y, y con una sétima en la base de la cola. En la fotografía de la izquierda se aprecia el iris color dorado.

se observó la coloración marrón, con el pecho y parte inferior de la cabeza color gris azulado, en los machos (Fig. 3) y dorada en el costado de las hembras durante la época reproductora (Bussing, 2002). Se depositaron muestras en el Museo de Zoología de la UCR con el número de registro UCR3297001.

A. septemfasciata se capturó en todos los muestreos y en los mismos ambientes que la especie anterior, para un total de 22 individuos. Si bien no queda claro si esta especie fue introducida en este río, sí es evidente que ya se han establecido poblaciones reproductivas que se han sumado a la ictiofauna nativa de la zona. Por lo tanto, se recomienda ampliar las investigaciones sobre esta especie, de manera que se permita ahondar en el conocimiento sobre su distribución natural.

Investigaciones como esta demuestran que queda mucho por conocer sobre la ecología de los peces de agua dulce, en especial en altitudes intermedias y ríos de zonas urbanizadas y ante los escenarios futuros de cambios climáticos globales. Con esta nota se espera contribuir al conocimiento sobre la fauna del Río Grande y de la distribución geográfica de estas tres especies. Se recomienda ampliar las investigaciones sobre la ictiofauna a mayores elevaciones de lo acostumbrado y sobre todo en áreas altamente impactadas por el crecimiento urbano.

Ética, conflicto de intereses y declaración de financiamiento: Los autores declaran haber cumplido con todos los requisitos éticos y legales pertinentes, tanto durante el estudio como en el manuscrito; que no hay conflictos de interés de ningún tipo, y que todas las fuentes financieras se detallan plena y claramente en la sección de agradecimientos. Asimismo, están de acuerdo con la versión editada final del documento. El respectivo documento legal firmado se encuentra en los archivos de la revista.

\section{AGRADECIMIENTOS}

Agradecemos a la Coordinación de Investigación de la Sede de Occidente y la Vicerrectoría de Investigación de la UCR por financiar el proyecto 540-B6-057 del cual se 

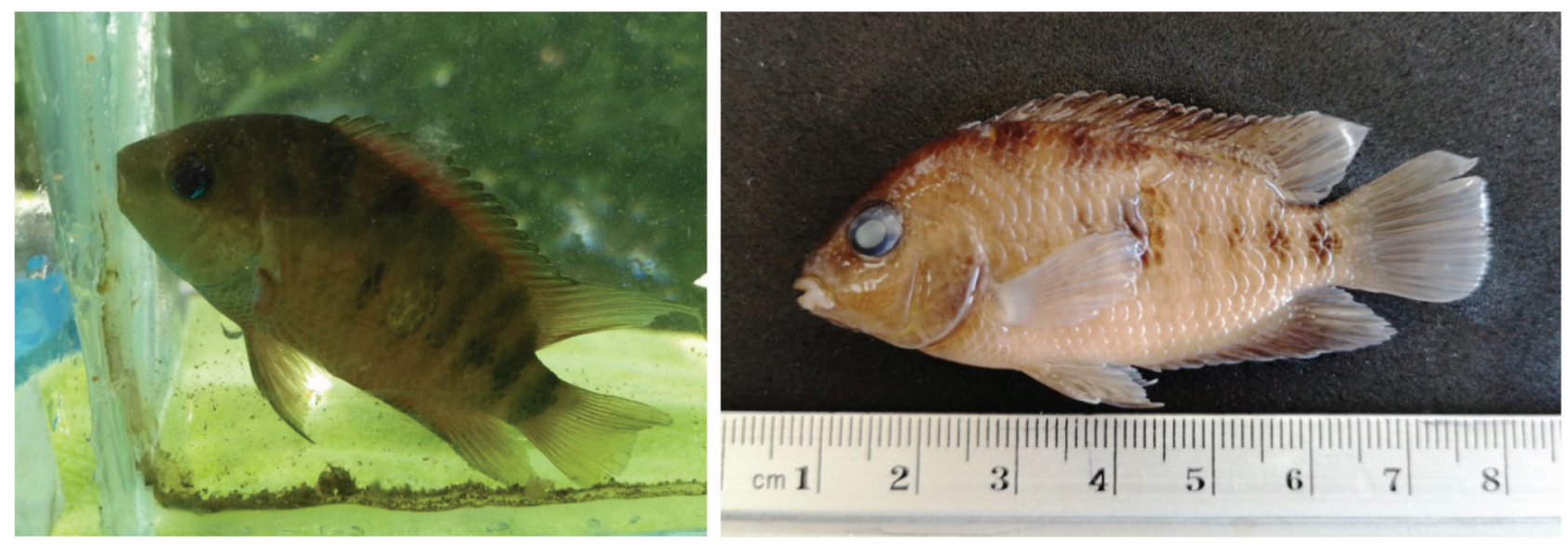

Fig. 3. Individuo de A. septemfasciata. Se observan como características diagnósticas: la mancha lateral ovalada en la tercera barra del patrón de seis barras y una sétima barra en el pedúnculo caudal. En la fotografía de la izquierda se aprecia el iris color azul característico.

obtuvieron los resultados presentados en este documento. A los asistentes que colaboraron en los muestreos: Juan Carlos Navarro y Adonay Mora, y a Arturo Angulo por corroborar la identidad de las especies.

\section{REFERENCIAS}

Angulo, A. (2013). Nombres comunes y técnicos de los peces de agua dulce de Costa Rica. Filología y Lingüística, 39(2), 77-103. DOI: 10.15517/rfl.v39i2.15061

Angulo, A., Garita-Alvarado, C., Bussing, W., \& López, M. (2013). Annotated checklist of the freshwater fishes of continental and insular Costa Rica: additions and nomenclatural revisions. Check List, 9(5), 987-1019. DOI: 10.15560/9.5.987

Bussing, W. (2002). Peces de aguas continentales de Costa Rica. San José, Costa Rica: Editorial de la Universidad de Costa Rica.

Fricke, R., Eschmeyer, W. N., \& Van der Laan, R. (Eds.). (2019). Eschmeyer's Catalog of Fishes: Genera, Species, References. Recuperado de http://researcharchive.calacademy.org/ research/ichthyology/catalog/fishcatmain.asp

Rodríguez, C., \& Zumbado, M. (2018). Informe final de investigación: Evaluación de la ictiofauna de la microcuenca alta de la Quebrada Estero en San Ramón de Alajuela.
Coordinación de Investigación de la Sede de Occidente de la Universidad de Costa Rica, San Ramón, Costa Rica.

Rojas, J., \& Rodríguez, O. (2008). Diversidad y abundancia ictiofaunística del río Grande de Térraba, sur de Costa Rica. Revista de Biología Tropical, 56(3), 1429-1447. DOI: $10.15517 /$ rbt.v56i3.5720 